\title{
AN EFFECTIVE, LOW-COST APPROACH TO IMPROVING MEDICATION ADHERENCE AMONG IMMIGRANT AND REFUGEE POPULATIONS
}

\author{
Ayobami Olanrewaju ${ }^{1}$, Maria Salguero ${ }^{1}$, Tetyana L. Vasylyeva ${ }^{1^{*}}$
}

Author information: ${ }^{1}$ Department of Pediatrics Texas Tech University Health Sciences Center, Amarillo, TX, USA; email: tetyana.vasylyeva@ttuhsc.edu

\begin{abstract}
Medication adherence among children is complex, requiring an adherent caregiver. Low educational level, low socioeconomic status and language barrier are factors influencing adherence. In presented case, the primary reason for non-adherence was the mother's poor communication skills due to a language barrier. We overcame the problem with a simple, virtually cost-free technique, which includes color labels and saw dramatic changes in medication adherence for our patient. This approach can be especially useful for immigrant and refugee populations, and we believe it can also complement existing interpretation services and other approaches to improve medication adherence for all patients.
\end{abstract}

Key words: Adherence, socioeconomics, children, refugee

CASE Our patient is a 6-month-old male with congenital nephrotic syndrome diagnosed at 10 -days-old. He requires periodic subcutaneous IgG infusions (done in the hospital as an outpatient procedure), and daily enoxaparin sodium (sub-cutaneous injection), enalapril, ranitidine, ferrous sulfate, citric acid, Poly- vi-sol ${ }^{\circledR}$, Indocin ${ }^{\circledR}$ (indomethacin), as well as high protein formula supplementation. His caregiver is an immigrant single mother, who speaks a rare Guatemalan-Spanish dialect. She speaks no English, and only completed primary level education, but she is determined to keep her child in good health. Her previous child died in infancy due to unknown medical conditions. The medical team tried different approaches to communicate with the mother about her infant's diagnosis and management with limited success. Hospital Spanish interpretation services were used for every encounter, Spanish-speaking residents were prioritized to the patient's care, and the Guatemalan embassy was contacted to improve communication. Home health nurses were provided to care for the patient and administer medications, but the nurses had problems communicating with the mother even when they were proficient in Spanish.

\footnotetext{
*Corresponding author: Tetyana L. Vasylyeva, Department of Pediatrics Texas Tech University Health Sci ences Center, Amarillo, TX, USA; email:

tetyana.vasylyeva@ttuhsc.edu
}

A Olanrewaju, M Salguero, Tetyana L.Vasylyeva(C
We eventually overcame the communication barrier to medication adherence using a simple, low-cost approach presented below. We call the approach "Label, Visualize, Time, and Train."

The first step was to identify the barriers to medication compliance. In our case, the mother understood that her child had a "kidney problem" and knew that he required daily medications, but she couldn't read labels and did not understand how to administer the medicines as prescribed. We used a summary page with visual cues that included a color code for each medication and each dosage level, the name of each drug, the dosing volumes and times (Figure 1). Colored tape was used to mark the drug containers and the dosage levels on a reference syringe for each drug. Syringes were used to measure the appropriate dose of each medication; the mother keeps the tape- marked syringes for reference and uses clean syringes for each dose. We trained the mother to use the simple method, and she was able to

proceed without difficulty.

Our approach, using a summary sheet that includes a color-code for the medication, the name of the drug, times to administer each medication, and the dose, effectively solved our communication problem. During the past 2 months, the mother has become confident in the care of her son with no further medication errors reported. Our patient has not required hospital admission since these changes were implemented and is doing well. This simple approach can be adapted for patients and caregivers with similar communication problems. 


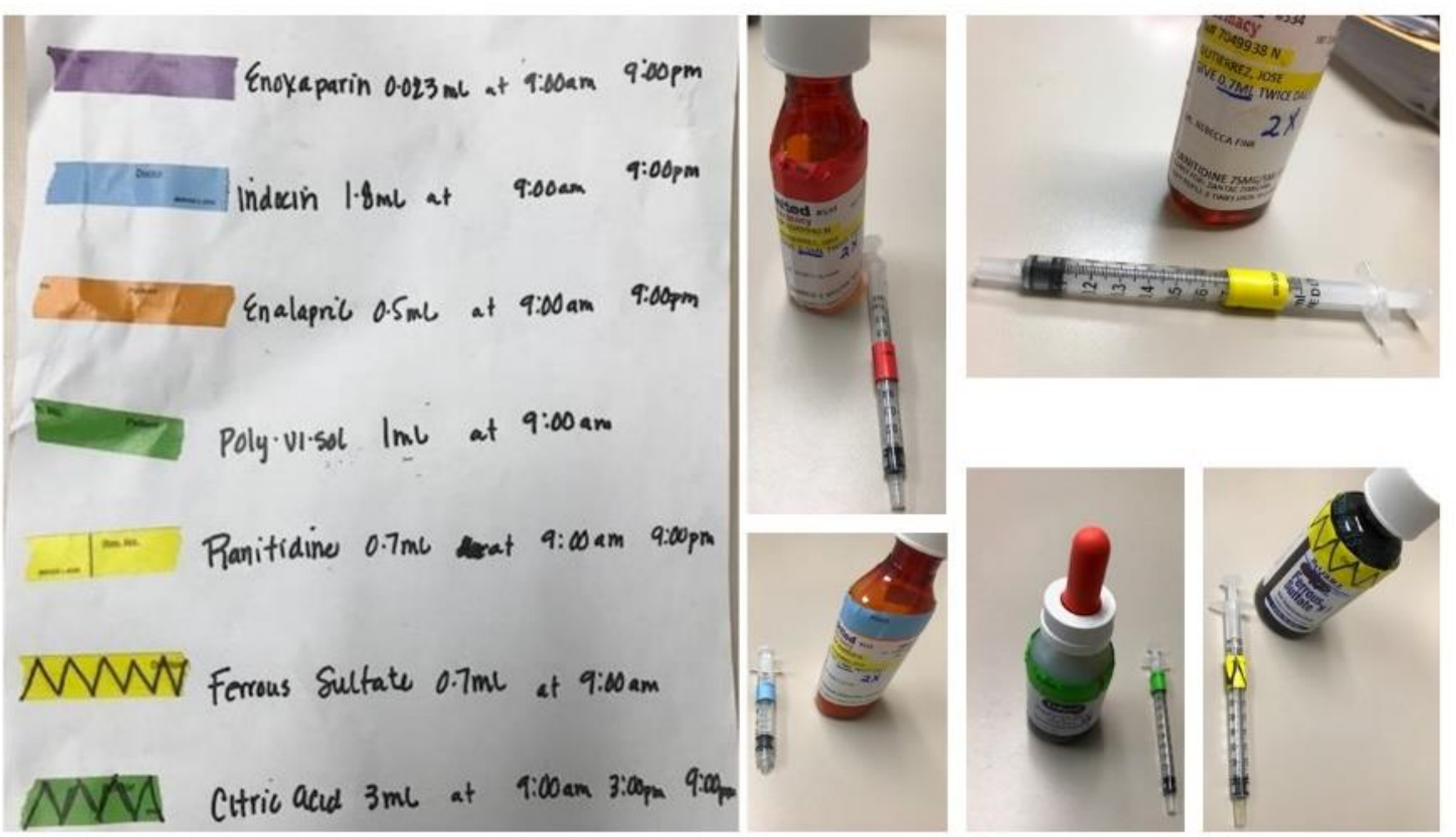

Figure 1. Visualization of marked medication.

DISCUSSION Medication adherence is a major problem in the care of patients with chronic illnesses. Patients with chronic illnesses who require long-term medications have adherence rates less than $50 \%$ with a further decline with time [1,2]. Poor medication adherence costs the health care system approximately $\$ 100$ billion annually, largely due to readmissions [1]. When questioned, the caregivers said that forgetfulness, other duties, confusion about instructions, and emotional issues were reasons for nonadherence [1].

Proper dosing for children is even more complex and requires a motivated, detail-oriented, and responsible adult to see to proper administration of medications to children [1]. Ramay et al. identified low educational level and low socioeconomic status as factors influencing adherence among children with chronic kidney diseases in Guatemala [2]. Patient education, improved dosing schedules, increased clinic hours (to ensure refills), and improved communication between patients and providers have been identified as key approaches to improve medication adherence [1]. A 2014 Cochrane review evaluating current approaches to improve medication adherence found that most approaches were complex and inefficient [3].

In our case, the primary reason for non-adherence was the mother's poor communication skills due to a language barrier. We overcame the problem with a simple, virtually cost-free technique and saw dramatic changes in medication adherence for our patient.

This approach can be especially useful for immigrant and refugee populations, and we believe it can also accompany existing interpretation services and other approaches to improve medication adherence for all patients.

We understand that this approach is by no means perfect, and some variants already exist that utilize specially designed pill bottles and tabs. However, to our knowledge, this is the first documented low-cost approach that can be applied by a primary care physician in rural areas without the need for specialized equipment.

\section{REFERENCES}

[1] Osterberg L, Blaschke T. Adherence to medication. N Engl J Med, 2005: 353: 487-97.

DOI: $10.1056 /$ NEJMra050100 
[2] Ramay BM, Cerón A, Méndez-Alburez LP. Lou-Meda R. Factors associated to acceptable treatment adherence among children with chronic kidney disease in Guatemala. PloS One, 2017; 12: e0186644.

DOI: 10.1371/journal.pone.0186644
[3] Nieuwlaat R, Wilczynski N, Navarro T, et al. Interventions for enhancing medication adherence. In: Nieuwlaat R editor. Cochrane Database of Systematic Reviews. Chichester, UK: John Wiley \& Sons; 2014. p. CD000011. DOI: 10.1002/14651858 